\title{
Nuclear matter within a dilatation-invariant parity doublet model: the role of the tetraquark at nonzero density
}

\author{
Susanna Gallas ${ }^{\mathrm{a}}$, Francesco Giacosa ${ }^{\mathrm{a}}$, and Giuseppe Pagliara ${ }^{\mathrm{b}}$ \\ ${ }^{a}$ Institute for Theoretical Physics, Johann Wolfgang Goethe University, \\ Max-von-Laue-Str. 1, D-60438 Frankfurt am Main, Germany and \\ ${ }^{b}$ Institut für Theoretische Physik, Ruprecht-Karls-Universität, \\ Philosophenweg 16, D-69120, Heidelberg, Germany
}

\begin{abstract}
We investigate the role of a scalar tetraquark state for the description of nuclear matter within the parity doublet model in the mirror assignment. In the dilatation-invariant version of the model a nucleon-nucleon interaction term mediated by the lightest scalar tetraquark field naturally emerges. At nonzero density one has, beyond the usual chiral condensate, also a tetraquark condensate. The behavior of both condensates and the restoration of chiral symmetry at high density are studied. It is shown that this additional scalar degree of freedom affects non negligibly the properties of the medium.

PACS numbers: 21.65.-f,12.39.Fe
\end{abstract}

\section{INTRODUCTION}

The properties of strongly interacting matter at finite baryon density have been widely investigated in the past by use of chiral models [1, 2]. This paper aims to study the effect of a light tetraquark field on such system. This subject is interesting for two reasons: (i) many works on light mesons spectroscopy show that the light scalar resonances below $1 \mathrm{GeV}$ listed in the PDG [3] can be successfully explained as a nonet of tetraquark states, see Refs. [4 7] and refs. therein. (ii) The nucleon can be modeled as a bound state of a quark and a (good) diquark 8]. It is then conceivable that two nucleons, in addition to the usual quark exchange leading to a quark-antiquark meson as intermediate state, interact via an exchange of a diquark, which leads to a tetraquark as mediator, see Fig. 1 for a pictorial representation.

In order to perform this study we use the linear sigma model for $N_{f}=2$ described in Ref. [9], in which the nucleon $N$ and its chiral partner $N^{*}$ form a baryon doublet, $\left(N, N^{*}\right)$, where $N^{*}$ is usually identified with the resonance $N(1535)$ [3]. The doublet is introduced in the so-called mirror assignment, first discussed in Ref. 10] and extensively analyzed in Refs. 9, 11, 12]. The particularity of the mirror assignment is the possibility to introduce a chirally invariant mass term $\sim m_{0}$, which does not originate from the quark condensate. In the framework of dilatation invariant interactions, this term originates from the condensation of two further scalar-isoscalar states: the dilaton/glueball field and the tetraquark field. In the present work we neglect the effect of the glueball since, due to its relatively high mass of about $1.5 \mathrm{GeV}$ [13], its exchange between nucleons is negligible in a first approximation. On the contrary, a light tetraquark state with a mass of about $600 \mathrm{MeV}$ and identified predominantly with the resonance $f_{0}(600)$ is potentially very interesting for the properties of nuclear matter. In addition, the usual (pseudo)scalar and (axial-)vector quarkantiquark mesons are present in the model.

Applications of the parity doublet model to nuclear matter and neutron stars were studied in Refs. [14-17] were it was shown that, at variance with the normal linear sigma model, it is able to describe the saturation of nuclear matter and it predicts a maximum mass for neutron stars compatible with observations. Some problems however arise from these studies: the nuclear matter compressibility turns out to be larger than the measured one and the value of $m_{0}$ needed to describe saturation is large, $\sim 800 \mathrm{MeV}$, and thus in disagreement with the results of the analysis of Ref. [9] in which a fit of vacuum properties resulted in $m_{0} \sim 500 \mathrm{MeV}$. Moreover the mass of the scalar-isoscalar meson responsible for the nucleon-nucleon attraction turns out to be very small, $m_{\sigma} \sim 350 \mathrm{MeV}$, a value that does not correspond to any particle in the PDG.

In the present study, we remove these inconsistencies of the parity doublet model between vacuum physics and finite density physics by regarding the resonance $f_{0}(600)$ as a predominantly tetraquark state and the resonance $f_{0}(1370)$ as the chiral partner of the pion, hence a predominantly quark-antiquark state. We show that nuclear matter saturation can be correctly reproduced with values for the compressibility compatible with data. The value of $m_{0}$ needed for fitting high density properties is the same of the one indicated by Ref. [9] in vacuum decays studies i.e. $m_{0} \sim 500$ $\mathrm{MeV}$. Quite remarkably, the need of having two scalars within the parity doublet model is in agreement with the implementation, in nucleon-nucleon potentials as the one of Bonn [18], of two scalar mesons having masses of $\sim 500$ and $\sim 1200 \mathrm{MeV}$.

The paper is organized as follows: in Sec. II we introduce the Lagrangian of the model, in Sec. III we obtain the corresponding thermodynamic potential in the mean field approximation, in Sec. IV we presents our results for nuclear matter and, finally, in Sec. V we draw our conclusions. 

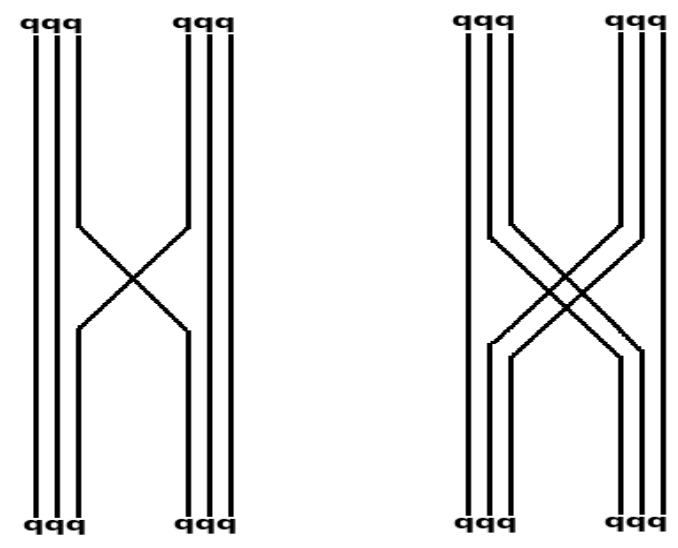

FIG. 1: The left picture represents the exchange of quarks between nucleons leading the nucleon-nucleon interactions mediated by quark-antiquark mesons. The right picture represents the exchange of diquarks leading to an additional contribution to the nucleon-nucleon potential mediated by tetraquark mesonic states.

Our units are $\hbar=c=1$, the metric tensor is $g^{\mu \nu}=\operatorname{diag}(+,-,-,-)$.

\section{THE PARITY DOUBLET MODEL IN THE BARYON SECTOR}

We present here the chirally symmetric linear sigma model with scalar, pseudoscalar, vector, axial-vector mesons, the nucleon and its chiral partner [9]. The scalar and pseudoscalar fields are included in the matrix

$$
\Phi=\sum_{a=0}^{3} \phi_{a} t_{a}=\left(\sigma+i \eta_{N}\right) t_{0}+\left(\vec{a}_{0}+i \vec{\pi}\right) \cdot \vec{t}
$$

where $\vec{t}=\vec{\tau} / 2$, with the vector of Pauli matrices $\vec{\tau}$, and $t^{0}=\mathbf{1}_{2} / 2$. Under the global $U(2)_{R} \times U(2)_{L}$ chiral symmetry, $\Phi$ transforms as $\Phi \rightarrow U_{L} \Phi U_{R}^{\dagger}$, where $U_{L}$ and $U_{R}$ are $2 \times 2$ unitary matrices. The vector and axial-vector fields are represented by the matrices

$$
V^{\mu}=\sum_{a=0}^{3} V_{a}^{\mu} t_{a}=\omega^{\mu} t^{0}+\vec{\rho}^{\mu} \cdot \vec{t}, \quad A^{\mu}=\sum_{a=0}^{3} A_{a}^{\mu} t_{a}=f_{1}^{\mu} t_{0}+{\overrightarrow{a_{1}}}^{\mu} \cdot \vec{t} .
$$

From these fields we define right- and left-handed vector fields $R^{\mu} \equiv V^{\mu}-A^{\mu}, L^{\mu} \equiv V^{\mu}+A^{\mu}$. Under global $U(2)_{R} \times U(2)_{L}$ transformations, these fields transform as $R^{\mu} \rightarrow U_{R} R^{\mu} U_{R}^{\dagger}, L^{\mu} \rightarrow U_{L} L^{\mu} U_{L}^{\dagger}$.

The identification of mesons with particles listed in the PDG [3] is as follows: the fields $\vec{\pi}$ and $\eta_{N}$ correspond to the pion and the $S U(2)$ counterpart of the $\eta$ meson, $\eta_{N} \equiv(\bar{u} u+\bar{d} d) / \sqrt{2}$, with a mass of about $700 \mathrm{MeV}$. This value can be obtained by "unmixing" the physical $\eta$ and $\eta^{\prime}$ mesons, which also contain $\bar{s} s$ contributions. The vector fields $\omega^{\mu}$ and $\vec{\rho}^{\mu}$ represent the resonances $\omega(782)$ and $\rho(770)$ and the axial-vector fields $f_{1}^{\mu}$ and ${\overrightarrow{a_{1}}}^{\mu}$ represent the resonances $f_{1}(1285)$ and $a_{1}(1260)$. Two possibilities for the identification of the $\sigma$ and $\vec{a}_{0}$ fields exist: $\left\{f_{0}(600), a_{0}(980)\right\}$ and $\left\{f_{0}(1370), a_{0}(1450)\right\}$. The first assignment is however unfavoured [19, 20] (for a general discussion of the issue of scalar mesons see also Refs. [21] and refs. therein), while the second is in agreement with the phenomenology.

The Lagrangian describing the meson fields is presented in detail in Ref. [9, 19]. For our purposes we notice that: (i) The chiral condensate $\sigma_{0}=\langle 0|\sigma| 0\rangle=Z f_{\pi}$ emerges upon spontaneous chiral symmetry breaking in the mesonic sector. The parameter $f_{\pi}=92.4 \mathrm{MeV}$ is the pion decay constant and $Z$ is the wave-function renormalization constant of the pseudoscalar fields [19, 22] and takes the value $Z=1.67 \pm 0.2$, which can be fixed from the process $a_{1} \rightarrow \pi \gamma$. (ii) In the case $N_{f}=2$ only one tetraquark state $\chi$ exists. It can be coupled to the model following Refs. [7, 23]. (iii) The dilaton/glueball field $G$ can also be easily added to the meson sector according to the requirement of dilatation invariance and the corresponding trace anomaly [20]. (For a general discussion of the glueball see Refs. 21, 24] and refs. therein.)

We now turn to the baryon sector, in which we have the baryon doublets $\Psi_{1}$ and $\Psi_{2}$, where $\Psi_{1}$ has positive parity and $\Psi_{2}$ negative parity. In the mirror assignment they transform as follows: 


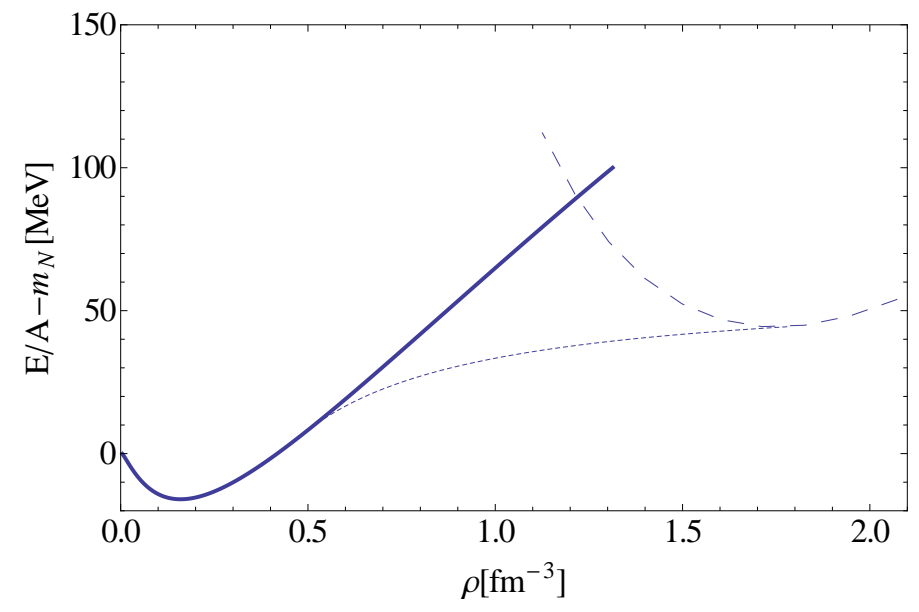

FIG. 2: Energy per baryon as a function of the density for the case $m_{0}=500 \mathrm{MeV}$ for the chiral symmetry broken solution (solid line) and the chiral symmetry restored solution (dashed line). The dotted line represent the Maxwell construction which matches the two solutions. The model can describe the saturation of nuclear matter.

$$
\Psi_{1 R} \longrightarrow U_{R} \Psi_{1 R}, \Psi_{1 L} \longrightarrow U_{L} \Psi_{1 L}, \Psi_{2 R} \longrightarrow U_{L} \Psi_{2 R}, \Psi_{2 L} \longrightarrow U_{R} \Psi_{2 L}
$$

i.e., $\Psi_{2}$ transforms in a "mirror way" under chiral transformations [10, 11]. These field transformations allow to write down a chiral baryonic Lagrangian:

$$
\begin{aligned}
\mathcal{L}_{\text {bar }} & =\bar{\Psi}_{1 L} i \gamma_{\mu} D_{1 L}^{\mu} \Psi_{1 L}+\bar{\Psi}_{1 R} i \gamma_{\mu} D_{1 R}^{\mu} \Psi_{1 R}+\bar{\Psi}_{2 L} i \gamma_{\mu} D_{2 R}^{\mu} \Psi_{2 L}+\bar{\Psi}_{2 R} i \gamma_{\mu} D_{2 L}^{\mu} \Psi_{2 R} \\
& -\widehat{g}_{1}\left(\bar{\Psi}_{1 L} \Phi \Psi_{1 R}+\bar{\Psi}_{1 R} \Phi^{\dagger} \Psi_{1 L}\right)-\widehat{g}_{2}\left(\bar{\Psi}_{2 L} \Phi^{\dagger} \Psi_{2 R}+\bar{\Psi}_{2 R} \Phi \Psi_{2 L}\right) \\
& -(a \chi+b G)\left(\bar{\Psi}_{1 L} \Psi_{2 R}-\bar{\Psi}_{1 R} \Psi_{2 L}-\bar{\Psi}_{2 L} \Psi_{1 R}+\bar{\Psi}_{2 R} \Psi_{1 L}\right)
\end{aligned}
$$

where $D_{1 R}^{\mu}=\partial^{\mu}-i c_{1} R^{\mu}, D_{1 L}^{\mu}=\partial^{\mu}-i c_{1} L^{\mu}$, and $D_{2 R}^{\mu}=\partial^{\mu}-i c_{2} R^{\mu}, D_{2 L}^{\mu}=\partial^{\mu}-i c_{2} L^{\mu}$ are the covariant derivatives for the nucleonic fields, with the coupling constants $c_{1}$ and $c_{2}$. The interactions of the baryonic fields with the scalar and pseudoscalar mesons are parametrized by $\widehat{g}_{1}$ and $\widehat{g}_{2}$. The last term in Eq. (4) generates a mass term when the tetraquark field $\chi$ and the glueball field $G$ condense:

$$
m_{0}=a \chi_{v a c}+b G_{v a c} .
$$

In Ref. [9] the quantity $m_{0}$ has been obtained through a fit procedure to known experimental and lattice quantities, obtaining:

$$
m_{0}=460 \pm 130 \mathrm{MeV} .
$$

In this paper we work under the simplified assumption $b=0$, i.e. the parameter $m_{0}$ is saturated by the tetraquark condensate and the corresponding nucleon-nucleon interaction is depicted in Fig. 1 right side. In this context the coupling constant $a$ is fixed as soon as the tetraquark condensate is specified, see next section. The physical fields $N$ and $N^{*}$ are related to the spinors $\Psi_{1}$ and $\Psi_{2}$ through:

$$
\Psi_{1}=\frac{1}{\sqrt{2 \cosh \delta}}\left(N e^{\delta / 2}+\gamma_{5} N^{*} e^{-\delta / 2}\right), \Psi_{2}=\frac{1}{\sqrt{2 \cosh \delta}}\left(\gamma_{5} N e^{-\delta / 2}-N^{*} e^{\delta / 2}\right) .
$$

The masses of the nucleons are obtained by diagonalizing the corresponding mass matrix in the Lagrangian. As function of the two condensates $\sigma_{v a c}$ and $\chi_{v a c}$ they read:

$$
m_{N, N^{*}}=\sqrt{\left(\frac{\widehat{g}_{1}+\widehat{g}_{2}}{4}\right)^{2} \sigma_{v a c}^{2}+\left(a \chi_{v a c}\right)^{2}} \pm \frac{\widehat{g}_{1}-\widehat{g}_{2}}{4} \sigma_{v a c} .
$$




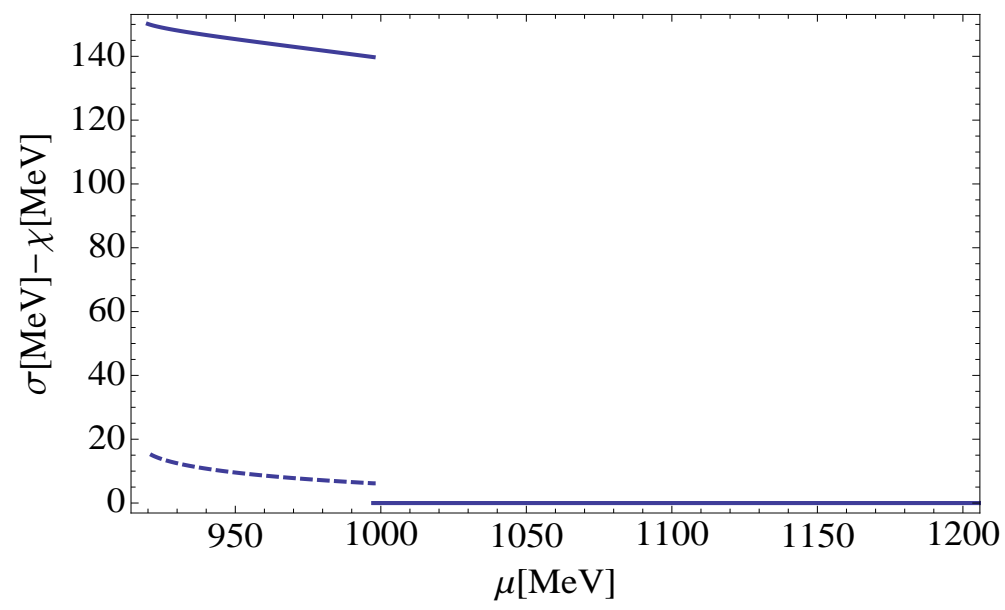

FIG. 3: Mean $\sigma$ (solid line) and $\chi$ (dashed line) fields. A strong first order phase transition is present at $\mu \sim 1 \mathrm{GeV}$. The transition point is the same for the two fields. Note, the minimal value for the chemical potential corresponds to nuclear matter.

\section{MEAN FIELD APPROXIMATION}

We now turn to the effective model in the mean field approximation suitable for describing nuclear matter. We thus retain only those fields which are relevant for such a study: the scalar fields $\sigma$ and $\chi$ and the vector field $\omega$. The effective Lagrangian is given by:

$$
\begin{aligned}
& \mathcal{L}_{e f f}=\frac{1}{2} \partial_{\mu} \sigma \partial^{\mu} \sigma+\frac{1}{2} \partial_{\mu} \chi \partial^{\mu} \chi-\frac{1}{4}\left(\partial_{\mu} \omega_{\nu}-\partial_{\nu} \omega_{\mu}\right)^{2} \\
& +\frac{1}{2} m^{2} \sigma^{2}+\frac{1}{2} m_{1}^{2} \omega_{\mu} \omega^{\mu}-\frac{1}{2} m_{\chi}^{2} \chi^{2}-\frac{\lambda}{4} \sigma^{4}+g \chi \sigma^{2}+\varepsilon \sigma \\
& +\bar{\Psi}_{1} i \gamma_{\mu} \partial^{\mu} \Psi_{1}+\bar{\Psi}_{2} i \gamma_{\mu} \partial^{\mu} \Psi_{2}-\frac{\widehat{g}_{1}}{2} \bar{\Psi}_{1} \sigma \Psi_{1}-\frac{\widehat{g}_{2}}{2} \bar{\Psi}_{2} \sigma \Psi_{2} \\
& -g_{\omega}^{(1)} \bar{\Psi}_{1} i \gamma_{\mu} \omega^{\mu} \Psi_{1}-g_{\omega}^{(2)} \bar{\Psi}_{2} i \gamma_{\mu} \omega^{\mu} \Psi_{2}+a \chi\left(\bar{\Psi}_{2} \gamma_{5} \Psi_{1}-\bar{\Psi}_{1} \gamma_{5} \Psi_{2}\right) .
\end{aligned}
$$

If we take their vacuum expectation values, $\sigma \rightarrow \sigma+\sigma_{v a c}$ and $\chi \rightarrow \chi+\chi_{v a c}$, the potential for the fields $\sigma$ and $\chi$ reads:

$$
V(\sigma, \chi)=\frac{1}{2}\left(\begin{array}{ll}
\chi & \sigma
\end{array}\right)\left(\begin{array}{cc}
m_{\chi}^{2} & -2 g \sigma_{v a c} \\
-2 g \sigma_{v a c} & m_{\sigma}^{2}
\end{array}\right)\left(\begin{array}{l}
\chi \\
\sigma
\end{array}\right)
$$

A non diagonal term, which is proportional to the parameter $g$ and mixes the quarkonium field $\sigma$ and the tetraquark field $\chi$, has emerged [30]. The physical fields, denoted as $h$ and $s$, are obtained through a standard diagonalization:

$$
\left(\begin{array}{l}
h \\
s
\end{array}\right)=\left(\begin{array}{cc}
\cos \theta_{0} & \sin \theta_{0} \\
-\sin \theta_{0} & \cos \theta_{0}
\end{array}\right)\left(\begin{array}{l}
\chi \\
\sigma
\end{array}\right), \theta_{0}=\frac{1}{2} \arctan \frac{4 g \sigma_{v a c}}{m_{\sigma}^{2}-m_{\chi}^{2}} .
$$

Then the physical masses of the scalar states are:

$$
\begin{aligned}
& m_{h}^{2}=m_{\chi}^{2} \cos ^{2} \theta_{0}+m_{\sigma}^{2} \sin ^{2} \theta_{0}-2 g \sigma_{v a c} \sin \left(2 \theta_{0}\right), \\
& m_{s}^{2}=m_{\sigma}^{2} \cos ^{2} \theta_{0}+m_{\chi}^{2} \sin ^{2} \theta_{0}+2 g \sigma_{v a c} \sin \left(2 \theta_{0}\right) .
\end{aligned}
$$

We identify here the predominately tetraquark state $h$ with $f_{0}(600)$ (the vacuum mass $m_{h}=600 \mathrm{MeV}$ is used) and the predominately quarkonium state $s$ with $f_{0}(1370)$ (the vacuum mass $m_{s}=1300 \mathrm{MeV}$ is used) . We now turn to the thermodynamic potential in the mean field approximation:

$$
\frac{\Omega}{V}=-\mathcal{L}_{\mathrm{M}}+\sum_{i} \frac{\gamma_{i}}{(2 \pi)^{3}} \int_{0}^{p_{F_{i}}} d^{3} p\left(E_{i}^{*}(p)-\mu_{i}^{*}\right)
$$




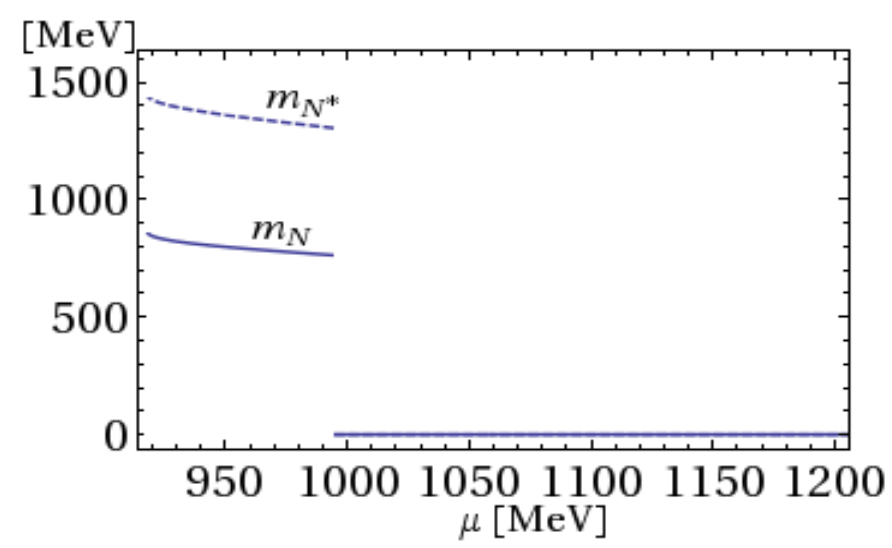

FIG. 4: Masses of the nucleons (solid line) and their chiral partners (dashed line) as function of the chemical potential.

where the mesons term reads

$$
\mathcal{L}_{\mathrm{M}}=\frac{1}{2} m_{1}^{2} \omega_{0}^{2}+\frac{1}{2} m^{2} \sigma^{2}-\frac{\lambda}{4} \sigma^{4}+\epsilon \sigma+g \chi \sigma^{2}-\frac{1}{2} m_{\chi}^{2} \chi^{2} .
$$

The index $i=N, N^{*}$ denotes the nucleon type (positive and negative parity nucleons), $\gamma_{i}=2 \times 2$ is the fermionic degeneracy (spin and isopsin), $p_{F_{i}}$ are the Fermi momenta, $E_{i}^{*}(p)=\sqrt{p_{i}^{2}+m_{i}{ }^{2}}$, and $\mu_{i}^{*}=\mu_{i}-g_{\omega} \omega_{0}=\sqrt{p_{F_{i}}^{2}+m_{i}{ }^{2}}$. The single particle energy of each parity partner $i$ is given by $E_{i}(p)=E_{i}^{*}(p)+g_{\omega} \omega_{0}$. The mean mesons fields are obtained by minimizing the thermodynamic potential, i.e. by solving the following system of non-linear equations:

$$
\begin{aligned}
& \frac{\partial(\Omega / V)}{\partial \sigma}=\lambda \sigma^{3}-m^{2} \sigma-\epsilon-2 g \chi \sigma+\rho_{N}^{*}\left(\sigma, \omega_{0}, \chi\right) \frac{\partial m_{N}}{\partial \sigma}+\rho_{N^{*}}^{*}\left(\sigma, \omega_{0}, \chi\right) \frac{\partial m_{N}^{*}}{\partial \sigma}=0 \\
& \frac{\partial(\Omega / V)}{\partial \omega}=m_{\omega}^{2} \omega_{0}-g_{\omega, N} \rho_{N}\left(\sigma, \omega_{0}, \chi\right)-g_{\omega, N^{*}} \rho_{N^{*}}\left(\sigma, \omega_{0}, \chi\right)=0 \\
& \frac{\partial(\Omega / V)}{\partial \chi}=-g \sigma^{2}+m_{\chi}^{2} \chi+\rho_{N}^{*}\left(\sigma, \omega_{0}, \chi\right) \frac{\partial m_{N}}{\partial \chi}+\rho_{N^{*}}^{*}\left(\sigma, \omega_{0}, \chi\right) \frac{\partial m_{N}^{*}}{\partial \chi}=0 .
\end{aligned}
$$

The scalar densities $\rho_{i}^{*}$ and the baryon densities $\rho_{i}$ are given by the expressions:

$$
\begin{gathered}
\rho_{i}^{*}=\gamma_{i} \int_{0}^{p_{F_{i}}} \frac{d^{3} \vec{p}}{(2 \pi)^{3}} \frac{m_{i}}{\sqrt{p_{i}^{2}+m_{i}^{2}}}=\frac{m_{i}}{\pi^{2}}\left[p_{F_{i}} E_{F_{i}}^{*}-m_{i}^{2} \ln \left(\frac{p_{F_{i}}+E_{F_{i}}^{*}}{m_{i}}\right)\right], \\
\rho_{i}=\gamma_{i} \int_{0}^{p_{F_{i}}} \frac{d^{3} \vec{p}}{(2 \pi)^{3}}=2 \frac{p_{F_{i}}^{3}}{3 \pi^{2}} .
\end{gathered}
$$

The parameters of the model are given by [19, 23]:

$$
\lambda=\frac{1}{2\left(Z f_{\pi}\right)^{2}}\left(m_{\sigma}^{2}-\frac{m_{\pi}^{2}}{Z^{2}}\right)+\frac{2 g^{2}}{m_{\chi}^{2}}, m^{2}=\frac{1}{2}\left(m_{\sigma}^{2}-3 \frac{m_{\pi}^{2}}{Z^{2}}\right), \varepsilon=\frac{f_{\pi} m_{\pi}^{2}}{Z}, a=\frac{m_{0} m_{\chi}^{2}}{g\left(Z f_{\pi}\right)^{2}} .
$$

Numerically one has $m_{\pi}=139 \mathrm{MeV}$ and $f_{\pi}=92.4 \mathrm{MeV}$. The parameter $Z$ is the wave function renormalization of the pseudoscalar fields [19, 22]. It is the only 'remnant' of the (axial-)vector mesons of the model. However, it should be stressed that the value of $Z=1.67$ strongly affects the properties of nuclear matter, for instance reducing the value of the chiral symmetry explicit breaking term, as we will show in the following. As already discussed in Refs. 19], the presence of (axial-)vector degrees of freedom in a chiral framework has non-negligible consequences on the (pseudo)scalar sector as well. The coupling constants of the nucleons and the scalar $\sigma$ meson are uniquely determined by the vacuum properties, $m_{0}=460 \pm 130 \mathrm{MeV}, m_{N_{\text {vac }}}=938 \mathrm{MeV}$ and $m_{N_{\text {vac }}^{*}}=1535 \mathrm{MeV}$ : $\widehat{g}_{1}=11.0 \pm 1.5, \widehat{g}_{2}=18.8 \pm 2.4[9]$. Finally, $g$ parametrizes the interactions of the tetraquark field with pions and the 


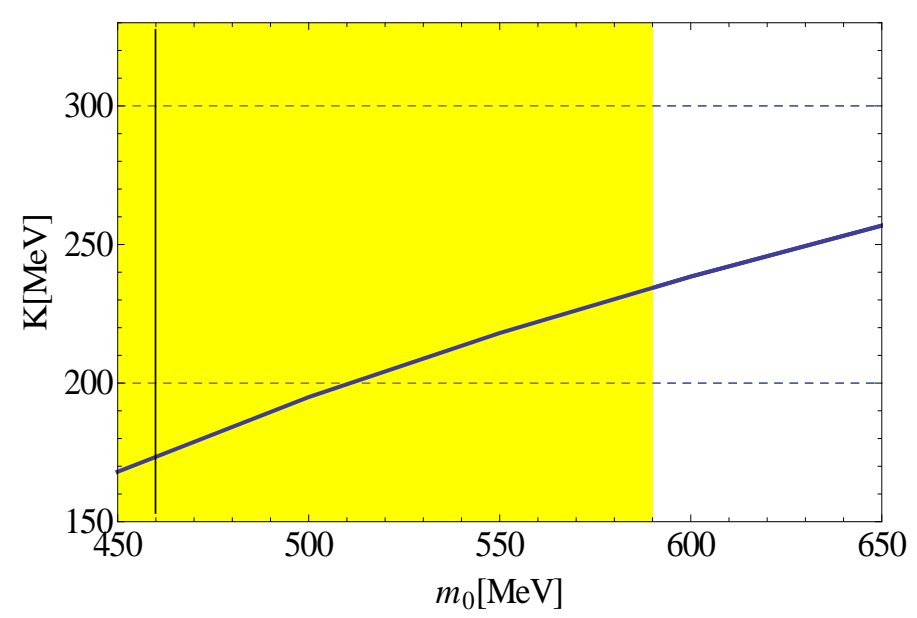

FIG. 5: Compressibility as a function of $m_{0}$ (solid line) and allowed range for $K$ as obtained by phenomenology (dashed lines). The constraint on the compressibility, together with vacuum decays properties, fixes $m_{0}$ to be in the range $500-600 \mathrm{MeV}$. The result $(460 \pm 130) \mathrm{MeV}$ is shown (gray (yellow online) band).

parameters $g_{\omega}^{(1)}, g_{\omega}^{(2)}$ describe the interactions of the $\omega$ meson with the nucleons. For sake of simplicity we assume that $g_{\omega}^{(1)}=g_{\omega}^{(2)}=g_{\omega}$ and thus, $g_{\omega, N}=g_{\omega, N^{*}}$. The quantities $g$ and $g_{\omega}$ are obtained from the following constraints of nuclear matter at saturation:

$$
\left.\frac{\partial}{\partial p_{F_{N}}}\left(\frac{E}{A}-m_{N_{v a c}}\right)\right|_{p_{F_{N}}=p_{F_{0}}}=0 \text { and }\left.\left(\frac{E}{A}-m_{N_{v a c}}\right)\right|_{p_{F_{N}}=p_{F_{0}}}=-16 \mathrm{MeV}
$$

where $E / A$ is the energy per nucleon and $p_{F_{N}}$ is the Fermi momentum of $N$. At the saturation point the latter equals value $p_{F_{N}}=p_{F_{0}}=258 \mathrm{MeV}$.

\section{RESULTS}

Let us fix $m_{0}$ to the intermediate value $m_{0}=500 \mathrm{MeV}$. From Eqs. (10) and (15) we can determine the four free parameters of the model which turn out to be: $g_{\omega}=4.87, g=450 \mathrm{MeV}, m_{\sigma}=1294 \mathrm{MeV}, m_{\chi}=612 \mathrm{MeV}$ (the latter two quantities generate the previously mentioned massed $m_{h}=600 \mathrm{MeV}$ and $m_{s}=1300 \mathrm{MeV}$ ). Quite remarkably the values of the masses of the two scalar mesons of our model are very close the values obtained within the "Bonn parametrization" of the nucleon-nucleon potential extracted by scattering data [18]. In Fig. 2, we show the energy per baryon $E / A$ as a function of the baryon density $\rho$ : one can notice the typical feature of the saturation of nuclear matter as a minimum of $E / A$ located at $\rho=\rho_{0}=0.16 \mathrm{fm}^{-3}$ and with a nucleon binding energy of $16 \mathrm{MeV}$.

The value of the compressibility, computed through the derivative of the pressure $P$ with respect to the density:

$$
K=\left.9 \frac{\partial P}{\partial \rho}\right|_{\rho=\rho_{0}}=194 \mathrm{MeV}
$$

a value which is very close to the standard range indicated by phenomenology $K=200-300 \mathrm{MeV}$ [25, 26]. In the figure we also display the energy per baryon for the chiral symmetry restored solution of the mean field equations (dashed line) and the interpolating Maxwell construction (dotted line) which we will explain in the following.

At high densities, our model predicts a strong first order chiral phase transition occurring at a chemical potential $\mu \sim 1 \mathrm{GeV}$ as displayed in Fig. 3 where we show the mean scalar mesons fields $(\sigma$ with solid line and $\chi$ with dashed line). The big jump in both the $\sigma$ and $\chi$ fields is computed by using a Maxwell construction: at a fixed value of the chemical potential the mean mesons fields are the solutions of Eqs. (11) which minimize the thermodynamic potential. In the energy per baryon - density diagram, the Maxwell construction corresponds to an hyperbolic branch (see Fig. 2, dotted line) given by the thermodynamic equation relating the pressure and the energy density: $e / \rho=\mu_{\text {crit }}-p_{\text {crit }} / \rho$ where $\mu_{\text {crit }}$ and $p_{\text {crit }}$ are the critical chemical potential and pressure. Notice that in absence of the tetraquark field and without the corrections related to $Z$ [15], the phase transition, while being still a first order, is much weaker. The parameter $Z=1.67$ indeed suppresses the chiral symmetry explicit breaking term and enhances the vacuum expectation value of $\sigma$, thus making the results for the chiral phase transition similar to the ones obtained in the 


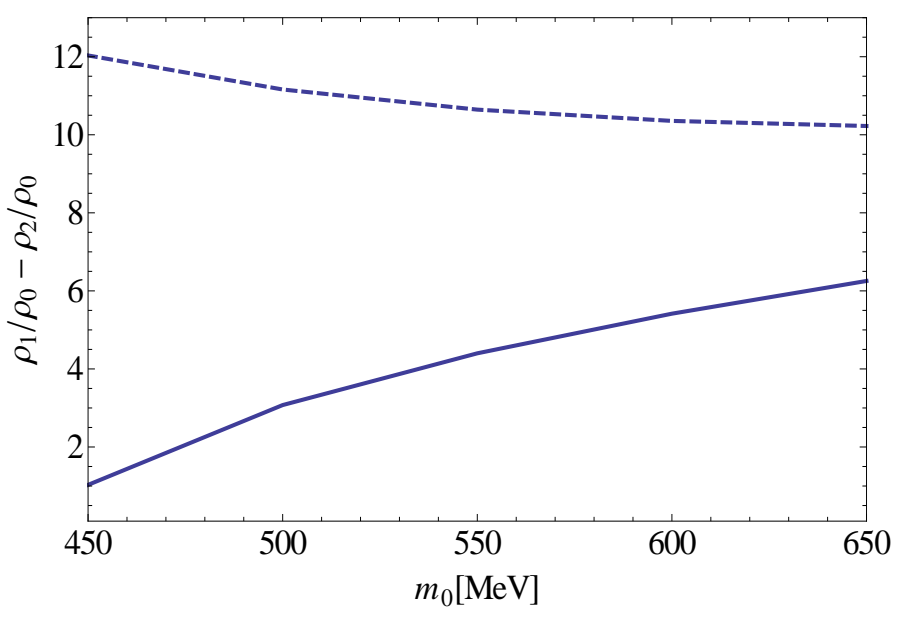

FIG. 6: Critical densities associated with the chiral phase transition as functions of $m_{0} \cdot \rho_{1}$ (solid line) corresponds to the onset of the phase transition and $\rho_{2}$ (dashed line) to the end (the densities are referred to the saturation density $\left.\rho_{0}\right)$. The first order phase transition becomes weaker for large values of $m_{0}$.

chiral limit. In our model the chiral phase transition is accompanied by the appearance of the chiral partners of the nucleon. While at low density only the nucleons are present, after the phase transitions both, the nucleons and their chiral partners, contribute the same amount. As one can notice in Fig. 4, in which we show the masses of the nucleons and their chiral partners as a function of the chemical potential, the nucleons become massless after the phase transition. This result is different from the one of Ref. [15] in which the nucleon mass, at high density, saturates to the fixed value of $m_{0}$. On the other hand, in our model, $m_{0}$ itself is a function of the density and at the chiral phase transtion it vanishes since the tetraquark condensate vanishes (see Eq. 5).

As a further step in our study we investigate the effect of varying the parameter $m_{0}$. For each value of $m_{0}$ we compute the values of the four parameters described before: while we can describe saturation for the whole range of Eq. (6), the value of $K$ turns out to be very small for small values of $m_{0}$. We show the corresponding result in Fig. 5 together with the range of $K$ indicated by phenomenology (dashed lines). By combing the information of Eq. (6) and the constraint obtained from the value of $K$ we can further restrict the range of the allowed values for the "bare mass" of the nucleon $500 \lesssim m_{0} \lesssim 600 \mathrm{MeV}$.

Finally, in Fig. 6] we show the values of the critical densities $\rho_{1}$ and $\rho_{2}$ which correspond to the onset and the end of the chiral phase transition. One can notice that the strength of the phase transition decreases for large values of $m_{0}$; within the allowed range of $m_{0}$ the density jump at the phase transition is anyway large : $\rho_{2} / \rho_{1}=2-4$. Remarkably, recent lattice results [27] show for a temperature $T \sim 0.8 T_{c}$ a rather broad range of density for the mixed phase, $\rho_{2} / \rho_{1} \sim 2$. Such studies are presently limited for temperatures $T \gtrsim 0.8 T_{c}$, therefore a quantitative comparison with our results is premature. Nevertheless, the lattice simulations seem to indicate that the interval for the mixed increase further when diminishing the temperature $T$. Future detailed analysis on this issue is surely interesting.

\section{CONCLUSIONS}

In this work we have used the chiral model developed in Ref. [9] to study nuclear matter properties. The model contains, in addition to the standard quark-antiquark fields with (pseudo)scalar and (axial-)vector quantum numbers, also a light scalar-isoscalar tetraquark field. In the framework of the mirror assignment the latter can be easily coupled to the nucleon by using the general requirement of dilatation invariant interactions and contributes to the nucleon-nucleon interaction, see Fig. 1.

Similarly to the results of Ref. [23] at finite temperature and vanishing density, we find that also at nonzero density a light tetraquark field has a strong influence on the medium properties of the system due to the interplay of two condensates, the tetraquark and the chiral (quark-antiquark) condensates. Interestingly, the described scenario and also the values of the coupling constants are in agreement with the work of Ref. [18], where two scalar fields are needed to describe nucleon-nucleon scattering data. As described in Ref. [28], in such a scenario nuclear matter is a prerogative of our world with three colors $\left(N_{c}=3\right)$, but would cease to exist as soon as a larger number of colors is considered.

An important parameter of the model is $m_{0}$, which describes the contribution to the nucleon mass which does not 
stem from the chiral condensate and which, in the present study, is saturated by the tetraquark condensate. Its value has been fixed in the vacuum as $m_{0}=460 \pm 130 \mathrm{MeV}$. It is then remarkable that the compressibility is in agreement with the experiment in a compatible range of $m_{0}$ (see Fig. 5), thus showing that vacuum results and nonzero density properties can be understood within the same theoretical model.

Further studies along the direction of the present work can be performed: (i) the contribution of the gluon condensate to $m_{0}$ and of the dilaton/glueball field to the nucleon-nucleon interaction should be included. Although the glueball is heavier $(\sim 1.5 \mathrm{GeV})$ and should not affect the interaction of two nucleons, its inclusion is important in a theoretical framework which is based on the dilatation invariance of QCD and on its anomalous breaking. (ii) The study of asymmetric nuclear matter and its application to neutron stars is also important since, recently, very massive neutron stars have been discovered which can give useful constraints for the stiffness of the equation of state. Moreover, also the symmetry energy of nuclear matter at high density is a crucial quantity that we can investigate by introducing in our model the scalar isovector $a_{0}(980)$, as done in Ref. [29], which in our assignment is also a tetraquark. (iii) The extension of the model to $N_{f}=3$ is currently under investigation. The effects of nonzero density and temperature of the complete model with all the relevant degrees of freedoms represents an interesting outlook of the present work.

The work of G.P. is supported by the Deutsche Forschungsgemeinschaft (DFG) under Grant No. PA 1780/2-1. We thank D.H. Rischke, J. Schaffner-Bielich and L. Bonanno for valuable discussions.

[1] N.K. Glendenning, Compact Stars, 2nd ed. 2000, Springer-Verlag, New York, Berlin, Heidelberg.

[2] I. Mishustin, J. Bondorf and M. Rho, Nucl. Phys. A 555 (1993) 215. P. Papazoglou, S. Schramm, J. Schaffner-Bielich, H. Stoecker, W. Greiner, Phys. Rev. C57 (1998) 2576-2588. P. Papazoglou, D. Zschiesche, S. Schramm, J. SchaffnerBielich, H. Stoecker, W. Greiner, Phys. Rev. C59 (1999) 411-427. L. Bonanno, A. Drago, A. Lavagno, Phys. Rev. Lett. 99 (2007) 242301. L. Bonanno, A. Drago, Phys. Rev. C79 (2009) 045801. E. K. Heide, S. Rudaz, P. J. Ellis, Nucl. Phys. A571 (1994) 713-732.

[3] K. Nakamura et al. (Particle Data Group), J. Phys. G 37, 075021 (2010).

[4] R. L. Jaffe, Phys. Rev. D15, 267 (1977). R. L. Jaffe, Phys. Rev. D15, 281 (1977).

[5] L. Maiani, F. Piccinini, A. D. Polosa, and V. Riquer, Phys. Rev. Lett. 93, 212002 (2004), hep-ph/0407017. G. 't Hooft, G. Isidori, L. Maiani, A. Polosa, and V. Riquer, Phys.Lett. B662, 424 (2008), 0801.2288. A. H. Fariborz, R. Jora and J. Schechter, Phys. Rev. D 72 (2005) 034001 arXiv:hep-ph/0506170. A. H. Fariborz, Int. J. Mod. Phys. A 19 (2004) 2095. arXiv:hep-ph/0302133. M. Napsuciale and S. Rodriguez, Phys. Rev. D 70 (2004) 094043.

[6] F. Giacosa, Phys. Rev. D74, 014028 (2006), hep-ph/0605191.

[7] F. Giacosa, Phys.Rev. D75, 054007 (2007), hep-ph/0611388.

[8] G. Hellstern, R. Alkofer and H. Reinhardt, Nucl. Phys. A 625 (1997) 697 arXiv:hep-ph/9706551. G. Hellstern, R. Alkofer, M. Oettel and H. Reinhardt, Nucl. Phys. A 627 (1997) 679 arXiv:hep-ph/9705267. M. Oettel, R. Alkofer and L. von Smekal, Eur. Phys. J. A 8 (2000) 553 arXiv:nucl-th/0006082. A. Bender, C. D. Roberts and L. Von Smekal, Phys. Lett. B 380 (1996) 7 arXiv:nucl-th/9602012. P. Maris and C. D. Roberts, Int. J. Mod. Phys. E 12 (2003) 297 arXiv:nucl-th/0301049. U. Vogl and W. Weise, Prog. Part. Nucl. Phys. 27 (1991) 195.

[9] S. Gallas, F. Giacosa, and D. H. Rischke, Phys.Rev. D82, 014004 (2010), 0907.5084.

[10] B. W. Lee, Chiral Dynamics (Gordon and Breach, New York, 1972).

[11] C. E. Detar and T. Kunihiro, Phys. Rev. D39, 2805 (1989).

[12] D. Jido, M. Oka, and A. Hosaka, Prog.Theor.Phys. 106, 873 (2001), hep-ph/0110005. D. Jido, Y. Nemoto, M. Oka, and A. Hosaka, Nucl.Phys. A671, 471 (2000), hep-ph/9805306.

[13] Y. Chen, A. Alexandru, S. Dong, T. Draper, I. Horvath, et al., Phys.Rev. D73, 014516 (2006), hep-lat/0510074.

[14] T. Hatsuda and M. Prakash, Phys. Lett. B224, 11 (1989).

[15] D. Zschiesche, L. Tolos, J. Schaffner-Bielich, and R. D. Pisarski, Phys.Rev. C75, 055202 (2007), nucl-th/0608044.

[16] V. Dexheimer, S. Schramm, and D. Zschiesche, Phys.Rev. C77, 025803 (2008), 0710.4192. V. Dexheimer, G. Pagliara, L. Tolos, J. Schaffner-Bielich, and S. Schramm, Eur.Phys.J. A38, 105 (2008), 0805.3301.

[17] C. Sasaki and I. Mishustin, Phys. Rev. C 82 (2010) 035204, 1005.4811.

[18] R. Machleidt, Phys.Rev. C63, 024001 (2001), nucl-th/0006014.

[19] D. Parganlija, F. Giacosa, and D. H. Rischke, Phys.Rev. D82, 054024 (2010), 1003.4934; D. Parganlija, F. Giacosa, and D. H. Rischke, AIP Conf.Proc. 1030, 160 (2008), 0804.3949; D. Parganlija, F. Giacosa, and D. H. Rischke, PoS CONFINEMENT8, 070 (2008), 0812.2183 [20]

[20] S. Janowski, D. Parganlija, F. Giacosa and D. H. Rischke, arXiv:1103.3238 [hep-ph].

[21] C. Amsler and N. A. Tornqvist, Phys. Rept. 389, 61 (2004). E. Klempt and A. Zaitsev, Phys. Rept. 454 (2007) 1 arXiv:0708.4016 [hep-ph]]. F. Giacosa, Phys. Rev. D 80 (2009) 074028 [arXiv:0903.4481 [hep-ph]].

[22] S. Struber and D. H. Rischke, Phys.Rev. D77, 085004 (2008), 0708.2389.

[23] A. Heinz, S. Struber, F. Giacosa, and D. H. Rischke, Phys.Rev. D79, 037502 (2009), 0805.1134.

[24] C. Amsler and F. E. Close, Phys. Rev. D 53 (1996) 295 arXiv:hep-ph/9507326. W. J. Lee and D. Weingarten, Phys. Rev. D 61, 014015 (2000). arXiv:hep-lat/9910008; F. E. Close and A. Kirk, Eur. Phys. J. C 21, 531 (2001). 
arXiv:hep-ph/0103173. F. Giacosa, T. Gutsche, V. E. Lyubovitskij and A. Faessler, Phys. Rev. D 72, 094006 (2005). arXiv:hep-ph/0509247. F. Giacosa, T. Gutsche, V. E. Lyubovitskij and A. Faessler, Phys. Lett. B 622, 277 (2005) arXiv:hep-ph/0504033. F. Giacosa, T. Gutsche and A. Faessler, Phys. Rev. C 71, 025202 (2005) arXiv:hep-ph/0408085. H. Y. Cheng, C. K. Chua and K. F. Liu, Phys. Rev. D 74 (2006) 094005 arXiv:hep-ph/0607206. Y. Chen et al., Phys. Rev. D 73 (2006) 014516. arXiv:hep-lat/0510074. V. Mathieu, N. Kochelev and V. Vento, Int. J. Mod. Phys. E 18 (2009) 1 arXiv:0810.4453 [hep-ph]].

[25] D. H. Youngblood et al., Phys. Rev. C69, 034315 (2004).

[26] C. Hartnack, J. Aichelin, and H. Oeschler, Int. J. Mod. Phys. D16, 153 (2007).

[27] A. Li, A. Alexandru, K. F. Liu and X. Meng, Phys. Rev. D 82 (2010) 054502 arXiv:1005.4158 [hep-lat]]. A. Li, A. Alexandru and K. F. Liu, arXiv:1103.3045 [hep-ph]. A. Li, A. Alexandru, X. Meng and K. F. Liu [chi QCD Collaboration], Nucl. Phys. A 830 (2009) 633C arXiv:0908.1155 [hep-lat]].

[28] L. Bonanno and F. Giacosa, arXiv:1102.3367] [hep-ph].

[29] B. Liu, V. Greco, V. Baran, M. Colonna and M. Di Toro, Phys. Rev. C 65 (2002) 045201 arXiv:nucl-th/0112034.

[30] The term responsible for the tetraquark-quarkonium interaction, and therefore also for the mixing, is $g \chi \sigma^{2}$ in Eq. (9). It was introduced in Ref. [7] because it is dominant in the large- $N_{c}$ expansion. (Terms of the kind $\chi^{2} \sigma^{2}$ would also possible, but they are here neglected because large- $N_{c}$ suppressed.) Note also that the term $g \chi \sigma^{2}$ is the only non-dilatation invariant term in the chiral limit. In a fully dilatation invariant context one should write down the term $\alpha G \chi \sigma^{2}$ where $G$ is the dilaton field. Upon condensation of the latter one obtains $g=\alpha G_{0}$. As long as the fluctuations of the dilaton are neglected it is justified to work with $g \chi \sigma^{2}$. 